\title{
常态化疫情防控下的仪器分析实验教学实践的探讨
}

\author{
林佳丽 1,2 , 王建 ${ }^{1, *}$, 陈珠灵 $1,2,{ }^{*}$, 袁耀锋 ${ }^{1}$ \\ 1 福州大学化学学院, 福州 350108 \\ 2 福州大学化学化工国家级实验教学示范中心，福州 350108
}

\begin{abstract}
摘要: 结合仪器分析实验的特点和教学中存在的问题, 阐述了虚拟仿真技术应用于仪器分析实验教学对线下实验的 补充作用, 并介绍了虚拟仿真实验教学在应对 “停课不停学” 等特殊状况的出色表现, 通过问卷和互动反馈, 准确 评估学生的学习状况, 指出虚拟仿真实验的局限性, 提出线上虚拟仿真与线下课堂实验相结合的教学模式, 促进仪 器分析实验教学质量的提升。
\end{abstract}

关键词: 虚拟仿真; 仪器分析实验; 线上教学; 教学改革

中图分类号: G64; O6

\section{Discussion on Experimental Teaching of Instrumental Analysis Under Normalized Epidemic Prevention and Control}

\author{
Jiali Lin ${ }^{1,2}$, Jian Wang ${ }^{1, *}$, Zhuling Chen ${ }^{1,2, *}$, Yaofeng Yuan ${ }^{1}$ \\ ${ }^{1}$ College of Chemistry, Fuzhou University, Fuzhou 350108, China. \\ ${ }^{2}$ National Experimental Teaching Demonstration Center for Chemistry and Chemical Engineering, Fuzhou University, \\ Fuzhou 350108, China.
}

\begin{abstract}
Based on the summary of the characteristics and existing problems of the instrumental analysis laboratory, the advantage of implementing virtual simulation technology was expounds. In addition, the outstanding performance of the virtual simulation laboratory teaching in dealing with special situations such as "classes suspended but learning continues" was introduced. Through questionnaires and interactive feedback, the learning situation of the students was evaluated. After pointing out the limitations of virtual simulation experiments, the teaching mode of combining online virtual simulation with offline classroom experiments was proposed, to promote the improvement of the quality of instrumental analysis laboratory teaching.
\end{abstract}

Key Words: Virtual simulation; Instrumental analysis laboratory; Online teaching; Teaching reform

仪器分析实验是化学专业本科生的必修课程之一, 与无机化学实验、有机化学实验等基础操作 实验课程相比, 仪器分析实验因其主要对象是价格昂贵的精密仪器而有自身的教学难点, 比如, 仪 器结构与工作原理等知识比较抽象、难以理解; 仪器台套数有限, 无法单人单套, 学生容易 “走马 观花”, 没有真正掌握实验操作等 ${ }^{[1,2]}$ 。虚拟仿真技术是网络技术迅猛发展的产物, 运用多媒体、人 工智能、虚拟现实、增强现实等先进的信息技术，营造出与真实实验场景、操作环节一致的软硬件

收稿: 2020-10-20; 录用: 2020-11-16; 网络发表: 2020-12-23

“通讯作者, Emails: jwang@fzu.edu.cn (王建); czl67@fzu.edu.cn (陈珠灵)

基金资助： 2018 年福建省本科高校重大教育教学改革研究项目(FBJG20180053)；2019 年福建省本科高校一般教育教学改革研究项目 (FBJG2019285); 2019 年福州大学一流本科教育教学改革研究项目(FDJG2019005); 福州大学一流本科教学改革建设重点项目 
环境 ${ }^{[3]}$ 。虚拟仿真技术因其资源呈现形式的多样性, 不受空间、时间、实验条件的限制等优点很大程 度上弥补了传统实验教学某些方面的缺憾, 丰富了传统的实验教学手段 ${ }^{[4-6]}$, 为仪器分析实验教学的 改革提供了方向。2020年春季学期, 为应对疫情对实验教学的影响, 我们开展了虚拟仿真技术在仪 器分析实验教学中的应用探索。

\section{1 仪器分析虚拟仿真实验对线下实验的补充作用}

在仪器分析实验的教学中, 了解仪器的结构和工作原理是十分重要的内容之一。虚拟仿真软件 每个项目加载了仪器介绍模块和实验原理模块, 以促进学生对仪器结构和仪器工作原理的理解。仪 器介绍模块通过三维动画模拟演示仪器的拆解和组装过程, 呈现仪器的组成部分和内部结构, 鼠标 点击各具体部件可显示其工作原理。实验原理模块链接了形象的图片和Flash动画, 展示抽象的理论 知识(如各个仪器主要元件的工作过程等)。

仪器分析实验的重点还包括仪器和软件操作。然而因仪器台套数有限, 学生线下实验难以真正 掌握仪器和软件操作。虚拟仿真实验系统的操作根据真实仪器以及真实实验数据作为支撑而编程设 计, 仪器操作步骤、软件操作界面、软件测试方法的编辑以及方法运行后触发的扫描信号变化等过 程都与真实情况非常接近, 非常适合进行实验操作的线上练习。

除了虚拟仿真外, 线上教学的其他资源, 比如教学视频、教学PPT等, 也可以通过拍摄教师现场 拆解仪器的主要部件, 同步讲解各部件的功能, 使学生深入了解仪器的内部构造和工作原理, 并通 过教师规范的实验操作演示, 帮助学生掌握实验操作。与上述资源相比, 虚拟仿真技术的优势在于 其直观性和趣味性。学生进入虚拟仿真实验系统, 需要通过操作完成一个与现实场景相似的实验过 程。如使用鼠标配制溶液, 观察、连接、调节仪器, 并使用仪器设备进行实验, 最后得到实验数据, 完成实验报告。学生通过这样的仿真操作, 能检验自身的学习效果, 了解操作过程中存在的问题, 教师也可通过后台系统直观地了解学生实验的自学情况, 从而调整教学重难点, 改进和充实教学内 容。

虚拟仿真技术，与其他传统的线上教学资源相互结合使用，共同对线下实验起到有效的补充， 辅助学生课前熟悉实验场景、自学实验内容, 课后反复练习、掌握仪器操作。

\section{2 疫情冲击下的仪器分析实验的开课探索}

2020年，一场突如其来、态势凶猛的新型冠状病毒肺炎疫情爆发了。为了阻断疫情向校园蔓延， 全国高校纷纷推迟2020年春季开学, 教育部发起 “停课不停学” 的号召, 鼓励高校结合实际开展线 上教学 ${ }^{[7]}$ 。有不少虚拟仿真实验教学平台组成联盟, 共同在疫情期间向全国高校免费提供实验教学 资源、软硬件资源、云资源等，保障高校在疫情防控期间的在线教学 ${ }^{[8,9]}$ 。

福州大学化学化工国家级实验教学示范中心在微瑞公司、欧贝尔公司的大力支持下, 从商品化 的虚拟仿真平台实验项目中, 优先挑选了仪器型号、实验内容与我校课程内容一致的项目作为教学 资源。在2020年春季学期的开学初, 将虚拟仿真实验开放给学生自主学习。进入期末, 由于疫情防 控的要求, 我校本科生仍无返校计划。在前期充分讨论协商的基础上, 仪器分析课程组决定本学期 进行线上授课, 采用虚拟仿真实验代替实验操作环节。

2020年春季学期仪器分析实验线上课程授课安排如表1所示, 我校开设的仪器分析实验包括高 效液相色谱仪、气相色谱仪、紫外-可见分光光度计、荧光分光光度计、傅里叶变换红外光谱仪、电 化学工作站、原子吸收分光光度计、电感耦合等离子体原子发射光谱仪、热重分析仪、X射线粉末衍 射仪、场发射透射电镜等 12种仪器对应的实验项目。前三周, 每周安排4天, 上午9:00-11:00进行具 体实验项目的线上直播授课, 后三周, 学生可自行安排时间完成虚拟仿真实验和实验报告。最后安 排8月17日进行本门课程的线上考试。 
大学化 学 Univ. Chem. 2021, 36 (9), 2010045 (3 of 8)

表1＼cjkstart仪器分析实验线上课程授课安排表

\begin{tabular}{ccc}
\hline 周次 & 日期 & 课程安排 \\
\hline $1-3$ & $6.29-7.19$ & 周一到周四9:00-11:00线上直播授课 \\
$4-6$ & $7.20-8.9$ & 完成虚拟仿真实验、提交实验报告 \\
8 & 8.17 & 线上考试 \\
\hline
\end{tabular}

为保证课程的顺利进行, 我们拟定了一系列实施细节:

第一, 建立仪器分析实验的 $Q Q$ 教学群, 便于师生交流、互动; 指导教师耐心细致地回答每一位 同学的疑问和困惑。

第二, 为每个学生和指导教师注册独立虚拟实验账号, 教师账号可查看学生登录虚拟仿真进行 实验操作的情况。

第三, 充分调动指导教师的积极性, 开课实验内容、仪器型号、实验要求不受原来实验教学大 纲、实验讲义约束, 解放思想, 大胆探索; 要求指导教师熟悉虚拟实验操作; 鼓励并协助教师拍摄 实验教学视频; 按计划通过腾讯课堂直播实验辅导讲座。

第四，对实验实施细节给出具体要求，在探索过程中，不断补充完善，仪器分析实验共72学时， 开设 12 个项目, 直播讲解时间 2 学时, 包括实验原理、虚拟或现场实操演示讲解。考虑到有不少同学 参加各高校的暑期夏令营, 各指导教师设置腾讯课堂具备回放功能, 方便学生自学。

第五, 充分理解学生的困难, 如: 留出较宽裕时间, 允许学生自己安排时间完成虚拟实验; 实 验报告采用电子版形式; 实验报告用的数据可以用仿真实验的数据, 也可用指导教师提供的数据。

第六, 仪器分析实验课程的考核, 线上笔试采用 “考试星” 与 “腾讯会议” 两款软件配合进行。 “考试星” 用于建立试题并发布给学生登录答题, 可支持多种题型共同组卷, 进行 “题目乱序” “选 项乱序” “页面切换自动交卷” 等防考场违规设置, 学生提交试卷后, 教师在系统后台即可获得学生 卷面、学生成绩、答题情况分析。“腾讯会议” 用于监考, 通过其视频功能, 全程保证考生面部双手 及桌面均处于监控范围。

第七，为保证成绩的合理性，采用结构成绩，包括腾讯课堂上线学时数、虚拟实验评判得分、 实验报告成绩、师生互动积极性、线上笔试成绩等, 综合评判期末成绩。确保总体成绩分布基本和 往年相当，有合理的区分度。

仪器分析实验线上教学流程如图1所示。

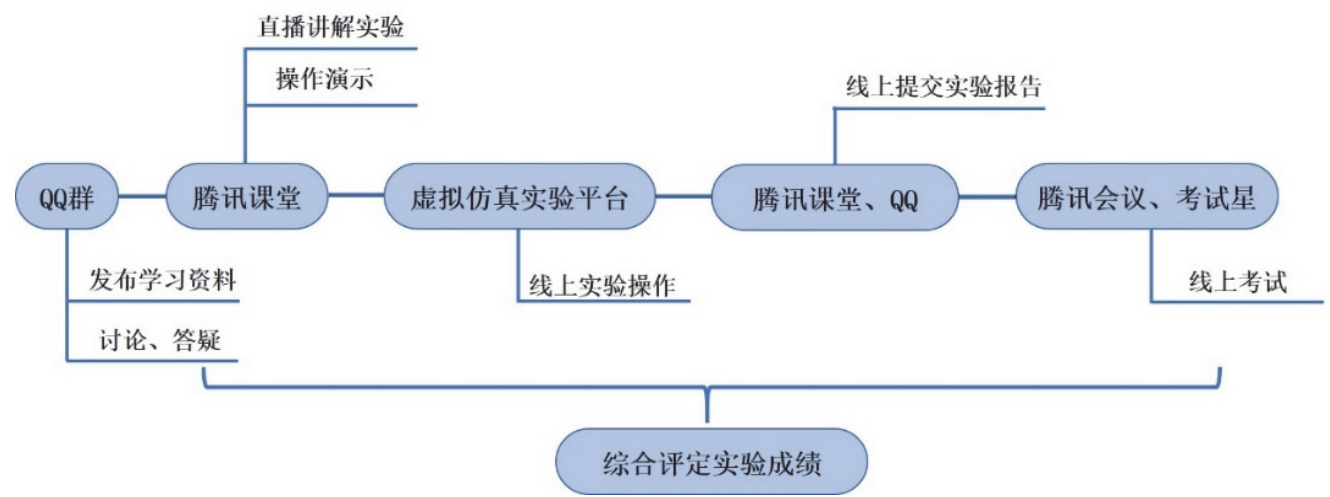

图1 仪器分析实验线上教学流程图

\section{3 线上教学实施效果与展望}

\section{1 虚拟仿真实验的自学效果}

我们通过线上问卷调查以及互动交流的形式对学生前期自学情况进行了总结。线上问卷的回答 
情况如图2所示, 虽然大多数 (83.67\%)学生对虚拟仿真这一新颖的实验教学模式感兴趣, 但在没有强 制要求的情况下, 仅有大约一半(45.94\%)的学生进行虚拟了仿真实验的体验, 说明虚拟仿真实验还 不足以调动学生的兴趣, 学生自主学习的积极性不高, 需要教师的监督和帮助。从进行虚拟仿真实 验学生的评价来看 $(25.23 \%$ 较满意, $11.71 \%$ 有帮助, $9.01 \%$ 不满意), 大部分学生能够从虚拟仿真这一 教学形式中获得较大的收获, 小部分学生则认为体验没有想象的好, 更希望在真实情境下完成实验。

关于开展线上虚拟仿真实验的征求意见

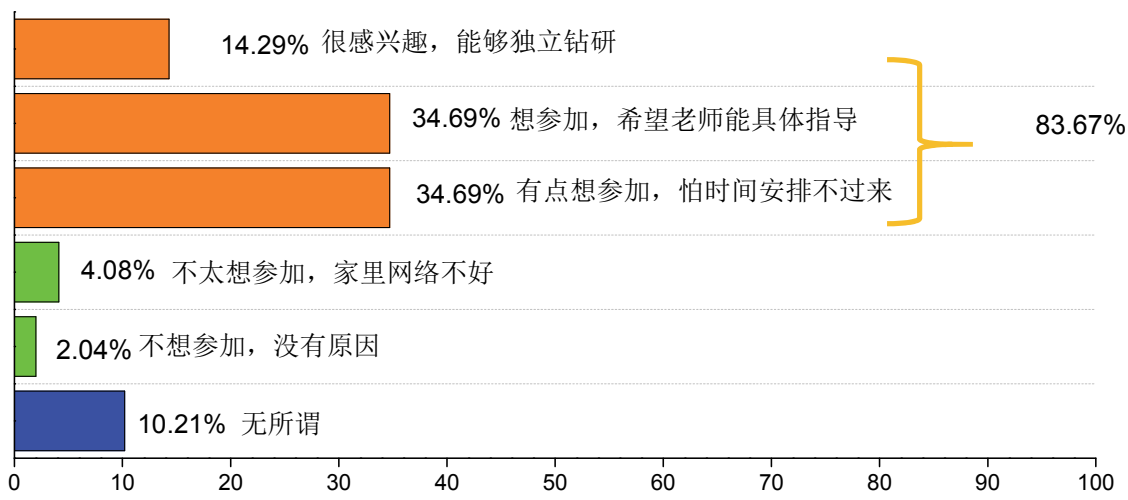

关于线上虚拟仿真实验学习情况的调研

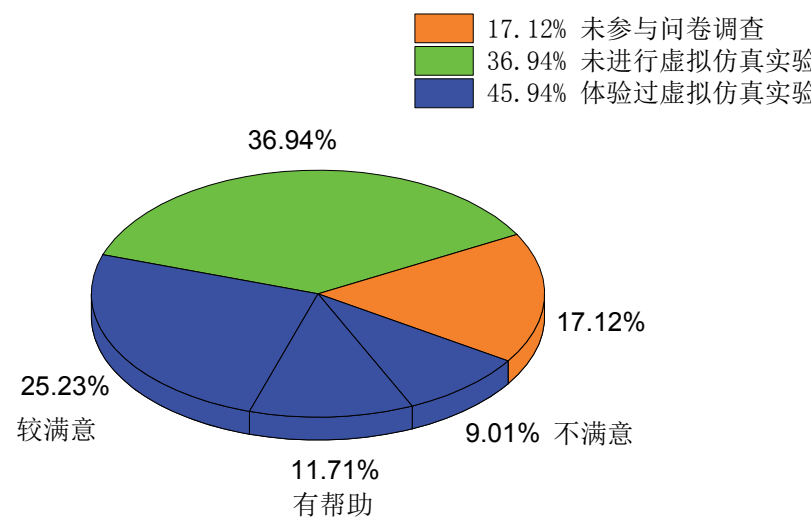

图2 虚拟仿真实验自学的问卷调查情况分析

\section{2 利用虚拟仿真进行实验线上教学的实施情况}

我们要求指导教师通过腾讯课堂加强对实验的直播讲解, 使虚拟仿真资源得到充分利用。从直 播课结束第二日对学生进行的仪器分析实验线上课程学习情况调查结果来看(图3), 大部分 $(82.54 \%)$ 学生表示除遇特殊情况外, 都会根据课程安排按时听课, 少部分(17.46\%)时间不能保证的学生也会 通过看回放的方式补课, $94.74 \%$ 的学生对疫情期间不得已采取的线上实验教学表示理解和接受。通 过直播讲解, $91.80 \%$ 的学生都能对虚拟仿真软件的操作流程和注意事项基本了解, 虽然课程安排了 宽裕的时间留给学生完成虚拟实验, 但接近一半的学生(49.18\%)跟随教师的讲解同步开展虚拟仿真 实验。说明通过前一阶段线上理论课程的学习 ${ }^{[10,11]}$, 学生对线上教学比较适应, 大部分学生对专业 实验课程的学习比较重视, 能够保持积极的学习态度, 合理安排时间及时完成课程学习任务。 


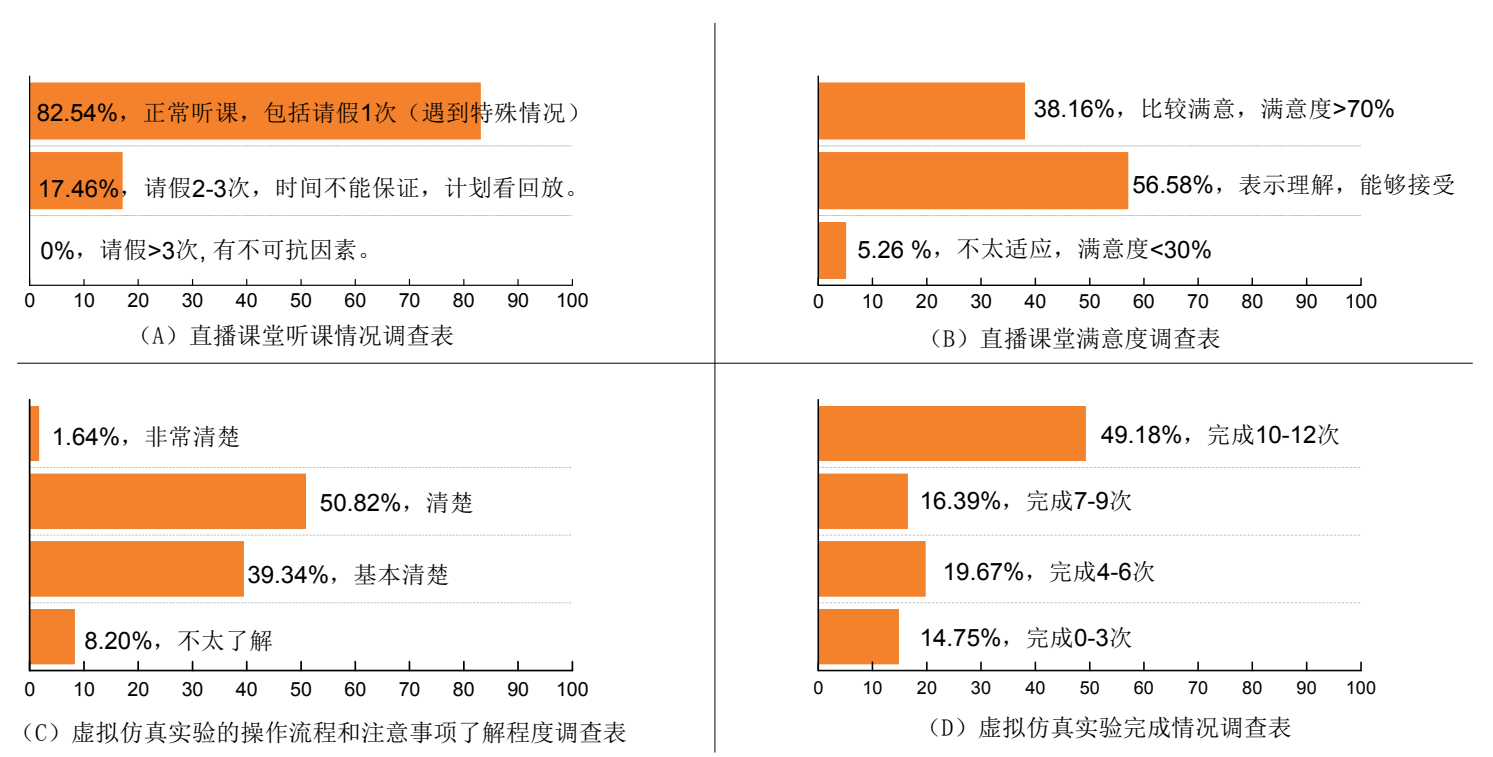

图3 仪器分析线上课程开展情况调查表

图4是2016级学生(2019年春季/线下实验)和2017级学生(2020年春季/线上课程)仪器分析实验的 成绩对比。考核方式上, 2019年春季线下课堂的成绩评价组成为平时成绩(课堂表现和实验报告)、期 末考试(笔试成绩和操作考试成绩), 2020年春季线上课程的成绩评价组成为平时成绩(直播课堂学习 情况、虚拟仿真操作得分、实验报告)、期末考试(仅笔试成绩)。笔试内容覆盖开设的全部实验内容, 主要考查基本理论、基本概念和基本技能。虽无法比较学生对仪器实际操作的掌握情况, 但从总体 成绩、平时成绩、笔试成绩的分布来看, 今年与去年差别不大, 绝大多数学生的平时成绩以及考试 成绩都在 80 分以上, 说明绝大多数学生能够较好地完成本门课程的学习任务。
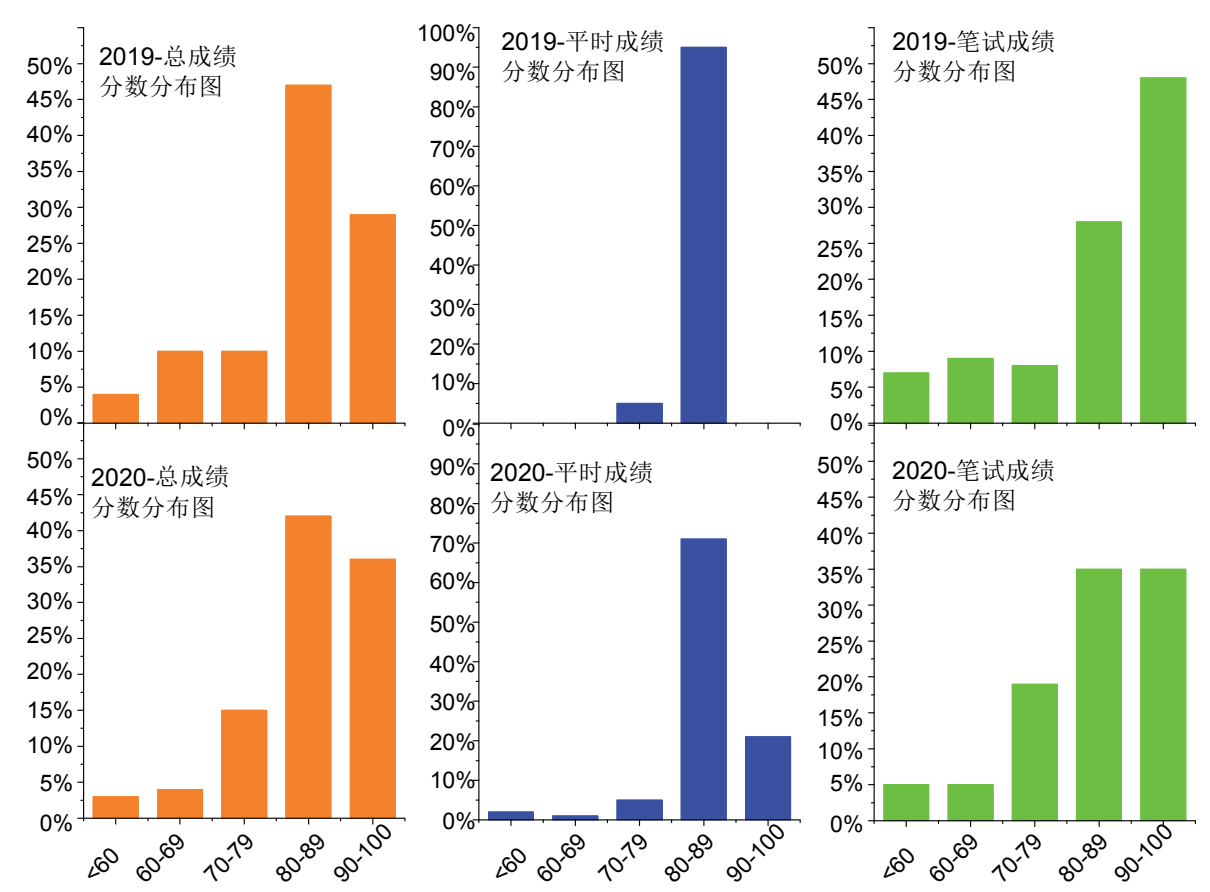

图4 线上线下实验学习成绩对比 
图5是学生对虚拟仿真软件辅助仪器分析实验学习的反馈表, 从数据可以看出, 对于虚拟仿真实 验在增加课堂趣味性以及助力掌握专业课程理论知识、仪器和软件操作步骤以及了解仪器组成与内 部构造方面, 均有超过 $85 \%$ 的学生认为有所帮助, 认可度较高。从选项上分析, 选择有一点帮助的 学生的占比最大, 分布在大约 $40 \%-60 \%$ 区间, 大于必不可少和较大帮助之和。可见大部分学生都认 同虚拟仿真实验对仪器分析实验的学习有一定的辅助作用, 但认为这种辅助作用比较有限。我们分 析其原因可能有以下几点: 首先, 本学期仪器分析实验是完全的线上教学, 学生在接受问卷调查时, 尚未返校进入实验室接触和使用仪器, 未能准确体会虚拟仿真实验对熟悉真实实验仪器的辅助作用; 其次, 在进行虚拟仿真软件实验时, 学生往往更重视实验操作的正确完成, 以获得较高的分数。是 否去点击和学习仪器介绍模块和实验原理模块, 并不影响学生虚拟仿真实验成绩, 如果没有认真使 用这些模块的功能, 可能影响相应功能的评价。评价结果同时表明, 在引导和有效督促学生利用虚 拟仿真各个模块的资源和功能进行学习方面, 教师还需在今后的教学实践中采取更多的措施。此外, 对提高分析和解决问题的能力以及对将来就业的帮助方面, 较大比例的学生认为帮助不大。

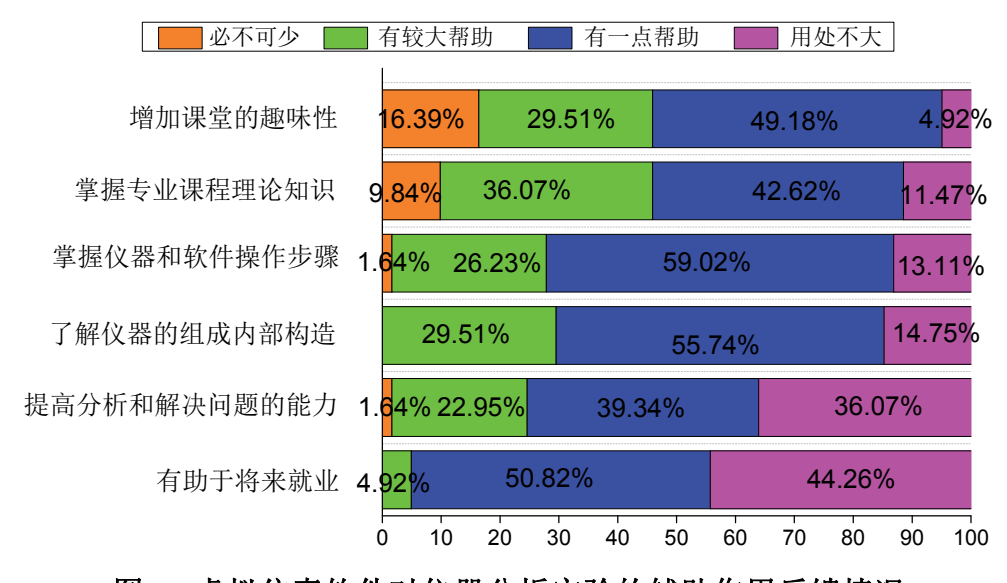

图5 虚拟仿真软件对仪器分析实验的辅助作用反馈情况

电子版为彩图

基于学生的评价反馈以及这段时间的教学实践, 我们也总结出线下课堂有着不可替代的优势。

首先, 线下课堂对现场解决问题能力以及实验动手能力的培养无可取代。虚拟仿真实验过程中 不会出现实际实验中可能出现的各种问题, 无助于解决问题能力的提升。同时, 线上虚拟仿真实验, 主要是鼠标操作, 无法对学生的基础实验操作技能进行训练。

第二, 线下课堂的真实性带来的真实感无可取代。虚拟仿真实验虽然在仪器操作、实验过程等 方面对真实环境都有较逼真的模拟, 但因为程序预设的限制, 实验过程过于理想化, 实验结果千篇 一律, 无法考查学生的个体差异。

第三, 虚拟仿真实验得不到真实数据, 不能从数据中发现问题。基于线下课堂实验的真实数据 展开分析和讨论, 可以对分析方法进行评价, 还可以引导学生根据实验结果反思实验过程中存在的 问题, 是否存在不规范的操作引入实验误差等, 这是实验教学中培养学生思考水平的重要环节, 而 这部分内容是虚拟仿真实验无法实现的。

第四, 虚拟仿真软件发展至今, 在操作不够流畅、硬件需求过高、过程设计过于简单和理想化 等方面仍有值得改进之处。

图6是学生对虚拟仿真实验和传统实验进行比较的情况, 大部分(54.67\%)学生认为虚拟仿真实验 对学习的帮助不如线下传统实验, 完全的线上教学让学生们非常遗憾没有机会熟悉现场仪器的使用。 针对这一情况, 秋季学期返校复课后, 我们安排了实习环节开放实验室, 进行预约上机操作训练, 做 
好虚拟实验和线下实操的衔接。

此外, 我们可喜地看到, 排除疫情防控的大背景, 更多的学生(57.14\%)对虚拟仿真和传统实验 相互补充相互结合的 “线上-线下”混合式教学表现出了期待。

(A) 虚拟仿真实验与传统实验, 哪个对你的学习更有帮助?

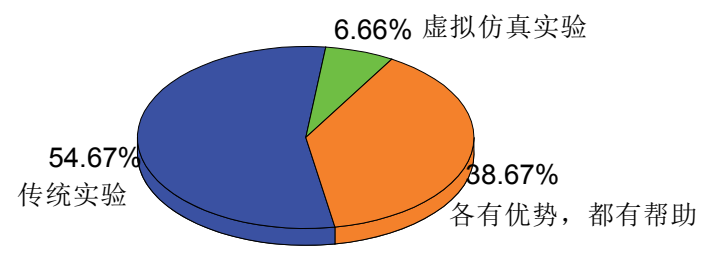

(B) 排除疫情防控大背景, 《仪器分析实验》课程 线上虚拟仿真实验是否可替代传统实验?

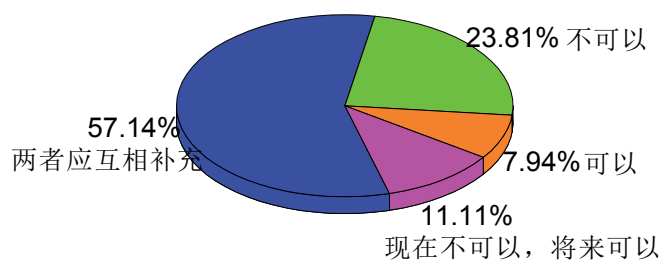

图6 虚拟仿真实验和传统实验进行比较的情况调查

\section{3 对 “线上-线下” 混合式教学的展望}

在今后的仪器分析实验教学中, 可充分利用本次虚拟仿真线上教学的探索经验和资源, 尝试线 上线下相结合, 探索最大化发挥虚拟仿真软件对线下实验辅助作用的方式, 丰富仪器分析实验内容, 提升仪器分析实验教学质量。

第一, 在进入实验室之前, 通过要求学生完成一个完整的虚拟仿真实验项目, 让学生熟悉实验 场景, 了解安全注意事项, 学习和掌握仪器的基本工作原理和使用方法。采取渐进式的教学策略, 在虚拟仿真实验项目中安排传统的经典的仪器分析实验, 线下课堂安排难度更高的实验, 完成更丰 富、更贴近生活的实际样品的检测。例如, 在紫外-可见分光光度法测定微量铁实验中, 虚拟仿真实 验项目可以设置现成的未知样中铁含量的测定, 在完成虚拟仿真实验的基础上, 线下课堂实验设置 实际样品菠菜中铁含量的测定实验; 在原子吸收分光光度法测定钙离子含量的实验中, 虚拟仿真实 验项目可以完成自来水中钻离子含量的测定, 线下课堂实验可以完成奶粉中钙含量的测定; 在电感 耦合等离子体发射光谱法的实验中, 虚拟仿真实验项目可以完成饮用水中金属元素的测定, 线下课 堂实验可以完成土壤或农产品中金属元素的测定。线上线下递进式的学习内容不会让学生认为是重 复实验而觉得枯燥, 反而带着兴趣和目的进行虚拟仿真的学习, 提高虚拟仿真学习的效果, 同时, 线下实验通过选取种类丰富的实际样品, 增加了仪器的实用性、实验的趣味性和项目的完整性。

第二, 通过虚拟仿真对仪器分析实验内容进行拓展和补充, 开展因设备昂贵或测试时间长等原 因限制实际教学开展的项目, 让学生接受更全面的实验训练, 实现本科教学与科研对接。例如增加 核磁共振波谱仪、扫描电镜等高成本、尚不能开设本科教学实验的大型贵重仪器的虚拟仿真线上实 验, 让学生熟悉现代化仪器表征手段; 增加耗时较长的复杂样品的前处理、仪器条件摸索和日常维 护等内容, 培养学生利用大型仪器解决复杂问题的综合能力和高级思维。

在受疫情影响的特殊时期, 学生无法真正走进实验室的情况下, 开展虚拟仿真实验, 不仅是缓 解因延期开学带来的教学压力的权宜之举, 同时也是对疫情过后进一步开展线上-线下融合的实验教 学改革的积极探索。

\section{4 结语}

虚拟仿真技术在仪器分析实验教学中对巩固基本理论、掌握仪器操作、熟悉实际应用等方面可 以起到积极的辅助作用。但在实际应用过程中, 不能仅依靠学生的自觉完成虚拟仿真实验, 教师的 监督和要求可以提高学生的完成效果, 使虚拟仿真资源得到充分利用。同时, 目前的化学类虚拟实 
验技术仍存在真实感不强、缺乏对实际实验中出现的各种问题的模拟、不能有效培养学生实际动手 操作能力和分析解决问题的能力等问题, 可以作为一种教学辅助手段和必要的补充, 而不能取代应 有的线下教学步骤。常态化疫情防控背景下, 我们将继续通过日常教学实践的不断探索, 摸索出更 加行之有效的线上虚拟仿真与线下课堂实验相结合的教学模式, 最大化地提升仪器分析化学实验教 学质量。我们也相信随着网络信息技术的发展, 虚拟仿真技术将不断推陈出新, 在仿真度、智能化 和实验设计上不断进步, 有望让学生体会如真实实验般的实验结果随环境、条件组合变化, 可探索、 发现、研究的实验乐趣。

\section{参 考 文 献}

[1] 高晓慧, 刘英姿, 刘浩然, 夏新华. 教育教学论坛, 2016, No. 43, 262.

[2] 马祥英, 陈其锋, 许海棠. 实验科学与技术, 2013, 11 (2), 76.

[3] 刘琼, 张韦深, 龙天澄, 汪雪兰. 中国医学教育技术, 2017, 31 (2), 162.

[4] 闵庆旺, 于长顺, 刘志广, 刘宇. 当代教育实践与教学研究, 2019, No. 7, 188.

[5] 彭敬东, 龚成斌, 马学兵, 张浩. 西南师范大学学报(自然科学版), 2017, 42 (7), 193.

[6] 白雁, 张娟, 潘瑾, 李永强. 实验技术与管理, 2011, 28 (12), 169 .

[7] 关于在疫情防控期间做好普通高等学校在线教学组织与管理工作的指导意见. [2020-02-05]. http://www.moe.gov.cn/jyb_xwfb/gzdt_gzdt/s5987/202002/t20200205_418131.html

[8] 化学实验免费在线教学平台--抗击新冠, 共克时艰. [2020-02-04]. https://mp.weixin.qq.com/s/ITWtUJXyf_P_FPC4Zn2T5A

[9] 停课不停学! 北京欧倍尔虚拟仿真学习平台免费开通, 邀您一起用知识武装自己! [2020-02-11]. http://www.bjoberj.com/html/about/news/2020/0211/472.html

[10] 袁耀锋, 林凌, 王建, 王心晨. 大学化学, 2020, 35 (5), 269.

[11] 朱亚先, 周立亚, 张树永, 郑兰䔉. 大学化学, 2020, 35 (5), 283. 